\title{
SMOOTHNESS PROPERTY FOR BIFURCATION DIAGRAMS
}

\author{
R. ROUSSARIE
}

\begin{abstract}
Strata of bifurcation sets related to the nature of the singular points or to connections between hyperbolic saddles in smooth families of planar vector fields, are smoothly equivalent to subanalytic sets. But it is no longer true when the bifurcation is related to transition near singular points, for instance for a line of double limit cycles in a generic 2-parameter family at its end point which is a codimension 2 saddle connection bifurcation point. This line has a flat contact with the line of saddle connections. It is possible to prove that the flatness is smooth and to compute its asymptotic properties.
\end{abstract}

\section{Structures of bifurcation diagrams}

Let $X_{\lambda}$ be a smooth $\left(\mathcal{C}^{\infty}\right)$ unfolding of planar vector fields, with parameter $\lambda \in \mathbf{R}^{k}$. In the parameter space $\mathbf{R}^{k}$, the bifurcation diagram $\Sigma$ is the set on which one has a variation of the topological type of $X_{\lambda}$, and inversely $X_{\lambda}$ is structurally stable for $\lambda \in \mathbf{R}^{k}-\Sigma$.

In the simpler situations, $\Sigma$ has a semi-algebraic structure (up to some differentiable diffeomorphism). For instance, for the codimension $k$ saddle-node unfolding, up to a smooth equivalence (which can be chosen $\mathcal{C}^{\ell}$ for an arbitrarily $\ell$ ), the unfolding is equivalent near $0 \in \mathbf{R}^{2}$, to:

$$
\pm y \frac{\partial}{\partial y}+\left(x^{k+1}+\sum_{i=0}^{k-1} a_{i}(\lambda) x^{i}\right) \frac{\partial}{\partial x}
$$

where $a_{i}(\lambda)$ are $\mathcal{C}^{\ell}$ functions $[\mathbf{D}]$. The bifurcation diagram is induced by the map $a(\lambda)=\left(a_{0}(\lambda), \ldots, a_{k-1}(\lambda)\right)$ from the semi-algebraic diagram for the roots of the polynomial $x^{k+1}+\sum_{i=0}^{k-1} a_{i} x^{i}$. 
As long as the bifurcations are related to analytic functions (up to smooth equivalences), the bifurcation diagram will have a sub-analytic structure (up to smooth diffeomorphisms). But in general, the bifurcation diagram will not be smoothly equivalent to a sub-analytic set. We want to discuss the more simpler situation where this phenomenon occurs, the generic saddle connection unfolding of codimension 2 . We suppose that $X_{\lambda}, \lambda \in \mathbf{R}^{2}$ is defined for $\lambda$ near 0 , and that $X_{0}$ has a hyperbolic saddle $s_{0}$ with a connection $\Gamma$ of some stable with some unstable separatrix.

Moreover, if $-\lambda_{1}(0), \lambda_{2}(0)$ are the eigenvalues of $s_{0}$ (with $\lambda_{1}(0)$, $\left.\lambda_{2}(0)>0\right)$, one supposes that $r(0)=\frac{\lambda_{1}(0)}{\lambda_{2}(0)}=1$.

Let $\sigma^{\prime}$ be some segment transverse to $\Gamma$ and $\sigma$ a half segment in $\sigma^{\prime}$, on the side of $\Gamma$ where a return map $P_{0}$ can be defined for $X_{0}$, from $\sigma$ to $\sigma^{\prime}$. Under the condition $r(0)=1$, the map $P_{0}$ is $\mathcal{C}^{1}$. As a first generic condition, one supposes that $P_{0}^{\prime}(0) \neq 1$ (we parametrize $\sigma$ by $x \in[0, X[$, 0 corresponding to $\sigma \cap \Gamma)$. Up to the change of $X_{\lambda}$ by $-X_{\lambda}$, one can suppose that $P_{0}^{\prime}(0)>1$ (expanding saddle connection). For $\lambda$ near 0 , one has a saddle point $s_{\lambda}$ of $X_{\lambda}$ near $s_{0}$ with eigenvalues $-\lambda_{1}(\lambda), \lambda_{2}(\lambda)$. Let be $r(\lambda)=\frac{\lambda_{1}(\lambda)}{\lambda_{2}(\lambda)}$ and $\alpha_{1}(\lambda)=1-r(\lambda)$.

Also, for $\lambda$ near 0 , the unstable separatrix near $\Gamma$ cuts $\sigma^{\prime}$ at a first point $b(\lambda)$ and the stable one at a first point $a(\lambda)$ (in the sequel, one chooses a smooth parametrization of $\sigma^{\prime}$ such that $\left.a(\lambda) \equiv 0\right)$. Let be $\alpha_{0}(\lambda)=b(\lambda)-a(\lambda)$.

As a second generic condition, one supposes that the smooth map $\lambda \rightarrow \alpha(\lambda)=\left(\alpha_{0}(\lambda), \alpha_{1}(\lambda)\right)$ is of maximal rank at $\lambda=0$ and so, one can suppose that $\lambda \equiv \alpha=\left(\alpha_{0}, \alpha_{1}\right)$. The bifurcation diagram is well understood [DRS1]. Of course the axis $\left\{\alpha_{0}=0\right\}$ is a line of saddle connection bifurcations. Next, one has an half line $D=\left\{\left(\alpha_{0}\left(\alpha_{1}\right), \alpha_{1}\right) \mid\right.$ $\left.\alpha_{1} \leq 0\right\}$, graph of some function $\alpha_{0}\left(\alpha_{1}\right)$ defined for $\alpha_{1} \leq 0$ and $\left|\alpha_{1}\right|$ small enough. Along this line one has a (double) semi stable limit cycle near $\Gamma$. The number of limit cycles is as follow: for $\alpha_{0}<0$ one has one unstable limit cycle. For $\alpha$ in the tongue between $\left\{\alpha_{0}=0\right\}$ and $D$, one has two limit cycles: a stable and an unstable one. These two limit cycles collide for $\alpha \in D$ and one has no limit cycle above $D$ (for $\alpha_{1} \leq 0$ ) and for $\alpha_{0} \geq 0, \alpha_{1} \geq 0$.

The function $\alpha_{0}\left(\alpha_{1}\right)$ is $\mathcal{C}^{\infty}$ for $\alpha_{1}<0$. It is easy to prove that there exist $A, B>0$ such that $\alpha_{0}\left(\alpha_{1}\right) \sim-B \alpha_{1} e^{A / \alpha_{1}}$ (see [DRS1]). Here and in the sequel the symbol $\sim$ means the equivalence of 1 -variable functions at $0\left(f(u) \sim g(u) \Longleftrightarrow f(u) / g(u) \rightarrow 1\right.$ for $u \longrightarrow 0_{ \pm}, \pm$depending on the case).

So the function $\alpha_{0}\left(\alpha_{1}\right)$ has a topological flat contact with $\left\{\alpha_{0}=0\right\}$, 
the line of saddle connections, and the diagram of bifurcation is not smoothly equivalent to an analytic one. Nevertheless we want to prove that $\alpha_{0}\left(\alpha_{1}\right)$ is smooth at $\alpha_{0}=0$. More precisely one has:

Theorem 1.1. The equivalence $\alpha_{0}\left(\alpha_{1}\right) \sim-B \alpha_{1} e^{A / \alpha_{1}}$ can be differentiated indefinitely.

Remark. As a consequence of the theorem $\frac{d^{\ell} \alpha_{0}}{d \alpha_{1}^{\ell}} \sim(-1)^{\ell+1} B \frac{A^{\ell}}{\alpha_{1}^{2 \ell-1}} e^{A / \alpha_{1}}$ for any $\ell \geq 0$. This implies that each derivative of $\alpha_{0}\left(\alpha_{1}\right)$ goes to 0 when $\alpha_{1} \rightarrow 0_{-}$. So, the function $\alpha_{0}\left(\alpha_{1}\right)$ is $\mathcal{C}^{\infty}$ at $\alpha_{1}=0$ (it is " $\mathcal{C}^{\infty}$-flat" at 0 ).

The precise value of $A, B$ are given in the paragraph 3 .

Related problems concern the bifurcations of zeros of abelian integrals. For instance, let us suppose that a smooth function $H$ on $\mathbf{R}^{2}$, has one saddle point $s$ and that the level of $s$ contains a saddle connection $\Gamma$.

Let be $\omega(m, \lambda)$ a smooth parameter family of 1-forms defined in some neighborhood of $\Gamma$.

Let be, as above a half segment $\sigma \simeq[0, X[$ transverse to the closed cycles of the function $H$ near $\Gamma$. We call $\gamma_{x}$ for $\left.x \in\right] 0, X$ [ the cycle through $x$.

Now, one considers the abelian integral:

$$
I(x, \lambda)=\int_{\gamma_{x}} \omega(m, \lambda) .
$$

It is easy to prove that $I(x, \lambda)=f(x, \lambda)-g(x, \lambda) \operatorname{Ln} x$ for some smooth functions $f, g$. If we suppose that $\omega(s, \lambda) \equiv 0$, then $g(0, \lambda) \equiv 0$ and the map $x \rightarrow I(x, \lambda)$ has a Dulac expansion at $x=0$ in the monomials $x^{i}$, $x^{i} \operatorname{Ln} x$, with smooth coefficients in $\lambda$ :

$$
I(x, \lambda)=\bar{\alpha}_{0}(\lambda)-\bar{\alpha}_{1}(\lambda) x \operatorname{Ln} x+\bar{\alpha}_{2}(\lambda) x+\cdots
$$

One looks at a singularity of codimension 2 of $I$, defined by $\bar{\alpha}_{0}(0)=$ $\bar{\alpha}_{1}(0)=0$. As a generic condition, one can suppose that the map $\lambda \rightarrow$ $\bar{\alpha}(\lambda)=\left(\bar{\alpha}_{0}(\lambda), \bar{\alpha}_{1}(\lambda)\right)$ is of maximal rank at $\lambda=0$ and that $\bar{\alpha}_{2}(0) \neq 0$. So that, one can suppose that $\lambda=\bar{\alpha}=\left(\bar{\alpha}_{0}, \bar{\alpha}_{1}\right)$ and also, by a smooth change of the parametrization, that $\bar{\alpha}_{2}(\bar{\alpha}) \equiv 1$.

The bifurcation diagram for $I(x, \bar{\alpha})$ is very similar to the above one, with a line $\bar{D}$ of double zeros for $I$, graph of a function $\bar{\alpha}_{0}\left(\bar{\alpha}_{1}\right)$ defined for $\bar{\alpha}_{1} \leq 0$, near 0 . We have now the same result of smoothness as above: 
Theorem 1.2. There exist constants $\bar{A}, \bar{B}>0$ such that $\bar{\alpha}_{0}\left(\bar{\alpha}_{1}\right) \sim$ $-\bar{B} \bar{\alpha}_{1} e^{\bar{A} / \bar{\alpha}_{1}}$ and this equivalence can be differentiated indefinitely.

The Theorem 1.2 can be seen as a limit case of Theorem 1.2. In fact the function $\delta(x, \lambda)=P(x, \lambda)-x$ (where $(P(x, \lambda)$ is the return map of $X_{\lambda}$ from $\sigma$ to $\sigma^{\prime}$ ) has a similar expansion as (1.1) where $-\operatorname{Lnx}$ is replaced by $\omega\left(x, \alpha_{1}\right)=\frac{x^{-\alpha_{1}}-1}{\alpha_{1}}$ (see $\left.[\mathbf{R}]\right)$, and one may notice that $\lim _{\alpha_{1} \rightarrow 0} \omega\left(x, \alpha_{1}\right)=-\operatorname{Ln} x$.

The abelian integrals are directly related to bifurcations of limit cycles for perturbations of hamiltonian vector fields, or dually for perturbations of integrable 1 -forms. Let be $\omega_{\varepsilon, \bar{\alpha}}$ a $(\varepsilon, \bar{\alpha})$-parameter family $(\varepsilon \in R)$, of smooth 1-forms,

$$
\omega_{\varepsilon, \bar{\alpha}}=d H+\varepsilon \nu_{\bar{\alpha}}+\circ(\varepsilon)
$$

where $\nu_{\bar{\alpha}}$ is an $\bar{\alpha}$-parameter family of 1 -forms. Then, the return map of $\omega_{\varepsilon, \bar{\alpha}}$ on a segment transverse to cycles of $H$, has the following expansion, in term of the hamiltonian value $h$ :

$$
P_{\varepsilon, \bar{\alpha}}(h)=h+\varepsilon \int_{\gamma_{h}} \nu_{\bar{\alpha}}+\circ(\varepsilon) .
$$

If $\bar{\alpha} \in \mathbf{R}^{2}$, one can define generic 3-parameter unfoldings, prescribing that $I(h, \bar{\alpha})=\int_{\gamma_{h}} \nu_{\bar{\alpha}}$ is a generic unfolding of abelian integrals.

One finds back for $\bar{\delta}(h, \bar{\alpha})=\left(P_{\varepsilon, \bar{\alpha}}(h)-h\right) / \varepsilon$ the situation of Theorem 1.1 when $\varepsilon \neq 0$ and of Theorem 1.2 when $\varepsilon=0$. In fact, one has a smoothness which is uniform in $\varepsilon$ :

Theorem 1.3. Take $\bar{\delta}(h, \bar{\alpha}, \varepsilon)=\frac{P_{\varepsilon, \bar{\alpha}}(h)-h}{\varepsilon}$. The bifurcation diagram of $\{\bar{\delta}=0\}$ has a surface of double zeros which is the graph of a smooth function $\bar{\alpha}_{0}\left(\bar{\alpha}_{1}, \varepsilon\right)$ defined above for $\varepsilon \geq 0, \bar{\alpha}_{1} \leq 0$. This function is $\mathcal{C}^{\infty}$-flat along the line $\left\{\bar{\alpha}_{0}=\bar{\alpha}_{1}=0\right\}$.

Remark. Perturbations of hamiltonian vector fields occur by blow-up of generic unfoldings. For instance the situation covered by Theorem 1.3 occurs in generic 3-parameter unfoldings to produce lines of saddle connections of codimension 2 (see [DRS1], [DRS2] for instance). The Theorem 1.3 shows that the diagram of bifurcation is smooth along these lines. So, for instance and as a consequence of Theorem 1.3, the bifurcation diagram of the cod. 3-Bogdanov-Takens bifurcation studied in [DRS1] is smooth. 
In the case of analytic families it would be interesting to obtain more informations on the bifurcation lines. For instance it is reasonable to hope that the line $\bar{D}$ would be defined by a Pfaffian function (in the sense of $[\mathbf{K}]$ ). Recent results about a preparation theorem for logarithmicexponential functions $[\mathbf{L R}]$, seem to be applicable to analytic unfoldings of abelian integrals to prove that the function $\bar{\alpha}_{0}\left(\bar{\alpha}_{1}\right)$ is a convergent series in powers of $\bar{\alpha}_{1}$ and $e^{1 / \bar{\alpha}_{1}}$. This would imply easily the Theorem 1.2 for analytic unfoldings. A similar result of convergence for Theorems 1.1 and 1.3 is more doubtful.

We begin by the proof of the Theorem 1.2 in the next paragraph. It is the easiest case because the transcendent function $\operatorname{Lnx}$ does not depend on the parameter. The proofs given for the Theorems 1.1 and 1.3 in the paragraphs 3 and 4 will follow the same general lines.

Anywhere in the text the symbol $\sim$ is for equivalence of functions of $x$ at $x=0$, or of $\alpha_{1}$ at $\alpha_{1}=0$.

\section{Smooth flatness in bifurcation diagrams of abelian integrals}

We will give a proof of Theorem 1.2 under some more general assumptions. Let be a function $\delta(x, \alpha)$ (we no longer write the bar subscript and we will write $\delta$ instead of $I)$, with parameter $\alpha=\left(\alpha_{0}, \alpha_{1}\right)$ near $0 \in \mathbf{R}^{2}$ and variable $x \in[0, X[$.

We will suppose that $\delta$ is smooth for $x \neq 0$ and has Dulac expansions at any order $\ell$ in $x, \operatorname{Lnx}$. This means that for any $\ell \in \mathbf{N}$, one can write:

$$
\delta(x, \alpha)=\sum_{0 \leq j \leq i \leq \ell+1} \alpha_{i j}(\alpha) x^{i} L n^{j} x+\Phi_{\ell}(x, \alpha)
$$

where the $\alpha_{i j}$ are smooth in $\alpha$ and $\Phi_{\ell}$ is a $\mathcal{C}^{\ell}$-function in $(x, \alpha)$ which is $\ell$-flat at $x=0\left(\Phi_{\ell}(0, \alpha)=\cdots=\frac{\partial^{\ell} \Phi_{\ell}}{\partial x^{\ell}}(0, \alpha)=0\right.$ for any $\left.\alpha\right)$.

Moreover one supposes that $\alpha_{00}(\alpha)=\alpha_{0}, \alpha_{11}(\alpha)=-\alpha_{1}$ and $\alpha_{10}(0) \neq$ 0 . Up to a change of variable depending on $\alpha$, and if necessary the change of $\delta$ in $-\delta$, one can suppose that $\alpha_{10}(\alpha) \equiv 1$. This will be assumed, from now on.

The set of parameters where $\delta$ has a double zero is given by:

$$
\left\{\begin{array}{l}
\delta=\alpha_{0}-\alpha_{1} x \operatorname{Ln} x+x+\cdots=0 \\
\frac{\partial \delta}{\partial x}=-\alpha_{1}(\operatorname{Ln} x+1)+1+\cdots=0
\end{array}\right.
$$


The equations (2.2) can be solved to give a germ of curve $\bar{D}=\left\{\left(\alpha_{1}(x)\right.\right.$, $\left.\left.\alpha_{2}(x)\right) \mid x \geq 0\right\}$ with:

$$
\alpha_{1}(x) \sim(\operatorname{Lnx})^{-1}
$$

and:

$$
\alpha_{0}(x) \sim-x(\operatorname{Ln} x)^{-1}
$$

It follows also from (2.2) that $\bar{D}$ is the graph of a function $\alpha_{0}\left(\alpha_{1}\right)$ for $\alpha_{1} \leq 0$ such that:

$$
\alpha_{0}\left(\alpha_{1}\right) \sim-e^{-1} \alpha_{1} e^{\frac{1}{\alpha_{1}}}
$$

We want to obtain similar equivalences for successive derivatives of $\alpha_{0}\left(\alpha_{1}\right)$, which are defined for $\alpha_{1} \neq 0$. More precisely we want to prove that the equivalence (2.5) can be indefinitely differentiated (and so, the function $\alpha_{0}\left(\alpha_{1}\right)$ will be smooth at $\left.\alpha_{1}=0\right)$.

The idea is to work with functions of $x$. So we postpone the study of $\alpha_{0}\left(\alpha_{1}\right)$ until the end of this paragraph and begin to say more about equations (2.2). First, we can solve the first equation to obtain a function $\widetilde{\alpha_{0}}\left(x, \alpha_{1}\right)$ :

Proposition 2.1. The equation $\delta=0$ defines a function $\alpha_{0}=$ $\widetilde{\alpha_{0}}\left(x, \alpha_{1}\right)$ where $\widetilde{\alpha_{0}}$ has Dulac expansions for any $\ell$ with smooth coefficients in $\alpha_{1}$. This means that there exists a sequence of smooth functions $\widetilde{\alpha}_{i j}\left(\alpha_{1}\right), 0 \leq j \leq i$, such that for any $\ell$ :

$$
\begin{gathered}
\widetilde{\alpha}_{0}\left(x, \alpha_{1}\right)=\sum_{0 \leq j \leq i \leq \ell+1} \widetilde{\alpha}_{i j}\left(\alpha_{1}\right) x^{i}(\operatorname{Ln} x)^{j}+\widetilde{\Phi}_{\ell}\left(x, \alpha_{1}\right) \\
\widetilde{\Phi}_{\ell}\left(x, \alpha_{1}\right), \mathcal{C}^{\ell} \text { and } \ell \text {-flat at } x=0 \text { for any } \alpha_{1} .
\end{gathered}
$$

Proof: For any $K \in \mathbf{N}$, one can write $\delta\left(x, \alpha_{0}, \alpha_{1}\right)=\Delta_{K}\left(x, x \operatorname{Ln} x, \alpha_{0}, \alpha_{1}\right)$ where $\Delta_{K}(u, v, w, z)$ is a $\mathcal{C}^{K}$-function.

Because $\frac{\partial \Delta_{K}}{\partial w}(0,0,0,0)=1$, one can solve $\Delta_{K}(u, v, w, z)=0$ as a $\mathcal{C}^{K}$-function $\widetilde{w}(u, v, z)$ near $(0,0,0)$ with $\widetilde{w}(0,0,0)=0$. If one takes in account that $\Delta_{K}(u, v, w, z)$ can be expanded in Taylor polynomials in $(u, v)$ with smooth coefficients in $(w, z)$, it turns out that $\widetilde{w}$ has Taylor polynomial of order $K$ in $u, v$, with smooth coefficients in $z$ :

$$
\widetilde{w}=\sum_{i, j \leq K} \widetilde{w}_{i j}(z) u^{i} v^{j}+\psi_{K}(u, v, z)
$$


where $\psi_{K}$ is $\mathcal{C}^{K}$ and $K$-flat at $(u, v)=(0,0)$, for any $z$.

To solve $\delta=0$, one has just to substitute $z=\alpha_{1}, u=x, v=x \operatorname{Ln} x$ in (2.7). Remark that $\psi_{K}\left(x, x \operatorname{Ln} x, \alpha_{1}\right)$ is $\mathcal{C}^{K-1}$ and $K-1$ flat in $x, \alpha_{1}$. To obtain the expansion at order $\ell$, it suffices to take $K=\ell+1$.

It is easy to obtain the first terms of $\widetilde{\alpha}_{0}$. One has:

$$
\widetilde{\alpha}_{0}\left(x, \alpha_{1}\right)=\alpha_{1} x \operatorname{Ln} x-x+\cdots
$$

where $+\cdots$ means a finite sum of terms as in (2.7) of higher order, plus a $\mathcal{C}^{\ell}$-flat function for an arbitrarily $\ell$.

Now we can substitute $\alpha_{0}=\widetilde{\alpha}_{0}\left(x, \alpha_{1}\right)$ in the second equation of $(2.2)$ to obtain an implicit equation for $\alpha_{1}(x)$.

$$
\delta_{1}\left(x, \alpha_{1}\right)=\frac{\partial \delta}{\partial x}\left(x, \widetilde{\alpha}_{0}\left(x, \alpha_{1}\right), \alpha_{1}\right)=0 .
$$

The function $\delta_{1}$ admits expansions as (2.6) at any order, but now with $j \leq i+1$. (This comes from the fact that $\delta_{1}$ is obtained by one differentiation in $x$.) The first terms in $\delta_{1}$ are:

$$
\delta_{1}\left(x, \alpha_{1}\right)=-(\operatorname{Ln} x+1) \alpha_{1}+1+\beta\left(\alpha_{1}\right) x(\operatorname{Ln} x)^{2}+\cdots
$$

for some smooth function $\beta$. The difficulty to solve the equation (2.9) is that $\frac{\partial \delta_{1}}{\partial \alpha_{1}}=-(\operatorname{Ln} x+1)+\cdots$ has a singularity at $x=0$. So, we will not try to solve it. We will keep $\alpha_{1}$ as an unknown function, and extend to negative powers of $x$ or $\operatorname{Ln} x$ the polynomial expressions we will consider.

Definition. $\mathcal{L}$ is the ring of smooth functions on $] 0, X[$ with the following properties. For any $f \in \mathcal{L}$ there exists a 2-variable function $F\left(x, \alpha_{1}\right)$ such that $f(x)=F\left(x, \alpha_{1}(x)\right)$.

For any $k$, this function $F$ can be written $F\left(x, \alpha_{1}\right)=F_{k}\left(x, \alpha_{1}\right)+$ $\Phi_{k}\left(x, \alpha_{1}\right)$ with:

1) $F_{k}\left(x, \alpha_{1}\right) \in \mathcal{C}^{\infty}\left(\alpha_{1}\right)\left[x, \operatorname{Ln} x, x^{-1},(\operatorname{Ln} x)^{-1}\right]$, i.e. $F_{k}$ is a polynomial in $x^{ \pm 1},(\operatorname{Ln} x)^{ \pm 1}$ with smooth coefficients in $\alpha_{1}$.

2) $\Phi_{k}\left(x, \alpha_{1}\right)$ is $\mathcal{C}^{k}$ and $k$-flat at $x=0$ for any $\alpha_{1}$.

3) $F_{k}$ is obtained by truncation of $F_{k+1}$.

Let $\mathcal{L}_{0} \subset \mathcal{L}$ be the subset of functions in $\mathcal{L}$ such that there exists a $k$ for which $F_{k}$ has a leading term $L(x)=x^{i}(\operatorname{Lnx})^{j} \not \equiv 1$ (i.e.: $i$ or $j \neq 0$ ) with coefficient $a\left(\alpha_{1}\right)$ with $a(0) \neq 0$.

We will also consider the ring $\mathcal{F}$ of fractions $f / g$ with $f \in \mathcal{L}$ and $g \in \mathcal{L}_{0}$. Any element in $\mathcal{F}$ can be written $\frac{f}{g}$ with $g=1+\cdots$ We define $\mathcal{F}_{0}$ as the set of fractions $\frac{f}{g} \in \mathcal{F}$ with $f \in \mathcal{L}_{0}$ and $g=1+\cdots$ 
Remark. Any element $h \in \mathcal{F}_{0}$ can be written $H\left(x, \alpha_{1}(x)\right)$ with $H\left(x, \alpha_{1}\right)=a\left(\alpha_{1}\right) L \frac{F}{G}$ with $F=1+\cdots, G=1+\cdots \in \mathcal{L}, a(0) \neq 0$ and $L(x)=x^{i}(\operatorname{Ln} x)^{j} \not \equiv 1$. It is clear that $h(x) \sim a(0) L(x)$ so $a(0)$ doesn't depend on the choice of $k$, if $k$ is large enough. On the contrary, the polynomial $F_{k}$ in the above definition is not uniquely defined. This follows from the fact that $\alpha_{1}(x)$ is implicit solution of (2.9). Nevertheless a formula as: $f=a L+\cdots$ will mean that $a$ and $L$ are the same for any $k$.

Lemma 2.2. $\alpha_{1}(x) \in \mathcal{F}_{0}$. More precisely:

$$
\alpha_{1}(x)=(\operatorname{Ln} x)^{-1} \frac{f}{1+(\operatorname{Ln} x)^{-1}} \quad \text { with } \quad f=1+\cdots \in \mathcal{L} .
$$

Proof: This is a direct consequence of the equation (2.9):

$$
\delta_{1}\left(x, \alpha_{1}\right)=-(\operatorname{Ln} x+1) \alpha_{1}+1+\cdots=0 .
$$

Clearly, it follows from Proposition 2.1 that the sum $f=1+\cdots$ belongs to $\mathcal{L}$ (in fact with monomials in $x, \operatorname{Lnx}$ ) and that $\alpha_{1}(x)=$ $(\operatorname{Ln} x)^{-1} \frac{f}{1+(\operatorname{Ln} x)^{-1}}$.

Lemma 2.3. $\mathcal{F}_{0}$ is closed by derivation: if $h \in \mathcal{F}_{0}$ then $\frac{d h}{d x} \in \mathcal{F}_{0}$. More precisely, if:

$$
h(x)=a\left(\alpha_{1}(x)\right) L(x) \frac{1+\cdots}{1+\cdots} \text { then } \frac{d h}{d x}=a\left(\alpha_{1}(x)\right) P\left(L^{\prime}\right) \frac{1+\cdots}{1+\cdots}
$$

where $P\left(L^{\prime}\right)=i x^{i-1}(L n x)^{j}$ if $L=x^{i}(L n x)^{j}$ with $i \neq 0$ and $P\left(L^{\prime}\right)=$ $j x^{-1}(\operatorname{Ln} x)^{j-1}$ if $L=(\operatorname{Ln} x)^{j}$.

Remark. If $L(x)=x^{i}(\operatorname{Lnx})^{j}, i$ or $j \neq 0$ :

$$
L^{\prime}(x)=\frac{d L}{d x}(x)=i x^{i-1}(\operatorname{Ln} x)^{j}+j x^{i-1}(\operatorname{Ln} x)^{j-1} .
$$

So $P\left(L^{\prime}\right)$ is the leading term of $L^{\prime}\left(P\left(L^{\prime}\right) \sim L^{\prime}\right)$.

Proof: Let be $f(x)=F\left(x, \alpha_{1}(x)\right) \in \mathcal{F}_{0}$ :

$$
f^{\prime}(x)=\frac{\partial F}{\partial x}\left(x, \alpha_{1}\right)+\frac{\partial F}{\partial \alpha_{1}}\left(x, \alpha_{1}\right) \frac{d \alpha_{1}}{d x} .
$$

We see that $\frac{d \alpha_{1}}{d x}$ enters in the expression (2.11). The function $\alpha_{1}$ itself belongs to $\mathcal{F}_{0}$ by Lemma 2.2 . So it is natural to begin the proof by this function. 
(a) The case of $\alpha_{1}$ :

Let us write again:

$$
\delta_{1}=-(\operatorname{Ln} x+1) \alpha_{1}+1+g\left(x, \alpha_{1}\right)
$$

with $g\left(x, \alpha_{1}\right)=\beta\left(\alpha_{1}\right) x(\operatorname{Ln} x)^{2}+\cdots$ as in (2.10). If we differentiate the equation $\delta_{1}=0$, we obtain:

$$
\left(-(\operatorname{Ln} x+1)+\frac{\partial g}{\partial \alpha_{1}}\right) \frac{d \alpha_{1}}{d x}=\frac{\alpha_{1}}{x}-\frac{\partial g}{\partial x} .
$$

Now, clearly enough, $\frac{\partial g}{\partial \alpha_{1}}$ and $\frac{\partial g}{\partial x} \in \mathcal{L}$ with:

$$
\frac{\partial g}{\partial \alpha_{1}}=\frac{d \beta}{d \alpha_{1}}\left(\alpha_{1}\right) x(\operatorname{Ln} x)^{2}+\cdots \text { and } \frac{\partial g}{\partial x}=\beta\left(\alpha_{1}\right)(\operatorname{Ln} x)^{2}+\cdots
$$

Multiplying $(2.12)$ by $(\operatorname{Lnx}+1)$ and taking in account that $(\operatorname{Lnx}+$ 1) $\alpha_{1}=1+\cdots \in \mathcal{L}$ one obtains:

$$
\left(-(\operatorname{Ln} x+1)^{2}+\cdots\right) \frac{d \alpha_{1}}{d x}=x^{-1}+\cdots
$$

So, the factor of $\frac{d \alpha_{1}}{d x}$ is in $\mathcal{L}_{0}$. If one put $x^{-1}(\operatorname{Lnx})^{-2}$ in factor, one has finally:

$$
\frac{d \alpha_{1}}{d x}=-x^{-1}(\operatorname{Ln} x)^{-2} \frac{1+\cdots}{1+\cdots}
$$

which belongs to $\mathcal{F}_{0}$. Remark that $-x^{-1}(\operatorname{Lnx})^{-2}$ is the derivative of $(\operatorname{Ln} x)^{-1}$, the leading term of $\alpha_{1} \sim(\operatorname{Lnx})^{-1}$.

(b) The general case:

To begin with, let us suppose that $h=f \in \mathcal{L}$, with $F\left(x, \alpha_{1}\right)=$ $a\left(\alpha_{1}\right) L(x)+\cdots, L(x)=x^{i}(\operatorname{Ln} x)^{j} i$ or $j \neq 0$. As above it is clear that $\frac{\partial F}{\partial x}\left(x, \alpha_{1}(x)\right)$ and $\frac{\partial F}{\partial \alpha_{1}}\left(x, \alpha_{1}(x)\right)$ belong to $\mathcal{L}$. Because $\frac{d \alpha_{1}}{d x} \in \mathcal{F}_{0}$ it follows that $f^{\prime} \in \mathcal{F}$. It remains just to check the leading term in (2.11). We have:

$$
\begin{aligned}
& \frac{d \alpha_{1}}{d x} \frac{\partial F}{\partial \alpha_{1}}=-\frac{\partial a}{\partial \alpha_{1}}\left(\alpha_{1}\right) x^{i-1}(\operatorname{Ln} x)^{j-2}+\cdots \\
& \frac{\partial F}{\partial x}\left(x, \alpha_{1}\right)=i a x^{i-1}(\operatorname{Ln} x)^{j}+\cdots \text { if } i \neq 0 \\
& \frac{\partial F}{\partial x}\left(x, \alpha_{1}\right)=j a x^{i-1}(\operatorname{Ln} x)^{j-1}+\cdots \text { if } i=0 .
\end{aligned}
$$


We see that in any case, the leading monomial comes from $\frac{\partial F}{\partial x}$. It is equal to a $P\left(L^{\prime}\right)$ as we want. If now $h=\frac{f}{g}$, with $f \in \mathcal{L}_{0}$, $g=1+\cdots \in \mathcal{L}$, it is easy to see that $h^{\prime}=\frac{f^{\prime}}{1+\cdots}$ and the result follows from the computation above.

We return now to the proof of the Theorem 1.2. To this end one introduces for any $k \geq 0$, the function $\varphi_{k}(x)=\frac{d^{k} \alpha_{0}}{d \alpha_{1}^{k}}\left(\alpha_{1}(x)\right)$, defined for $x \neq 0$.

We want to prove:

Proposition 2.4. For any $k, \varphi_{k} \in \mathcal{F}_{0}$ and:

$$
\varphi_{k}(x) \sim(-1)^{k+1} x(\operatorname{Ln} x)^{2 k-1} .
$$

Proof: $\varphi_{0}(x)=\alpha_{0}\left(\alpha_{1}(x)\right)=\alpha_{0}(x)$. Let us notice that equation (2.6) implies that $\alpha_{0} \in \mathcal{L}$ with expansion:

$$
\alpha_{0}=\alpha_{1} x \operatorname{Ln} x-x+\cdots
$$

Unfortunately $\alpha_{0}$ belongs to $\mathcal{L}$ with the bad coefficient $\alpha_{1}$ for the leading term. To prove that $\alpha_{0} \in \mathcal{F}_{0}$, we use the implicit equation (2.9) for $\alpha_{1}$ which gives:

$$
\alpha_{1}(\operatorname{Ln} x+1)=1+O\left(x(\operatorname{Ln} x)^{2}\right) \in \mathcal{L} .
$$

If one multiplies $(2.15)$ by $(\operatorname{Lnx}+1)$, one obtains:

$$
(\operatorname{Lnx}+1) \alpha_{0}=\alpha_{1}(\operatorname{Ln} x+1) x \operatorname{Ln} x-x(\operatorname{Ln} x+1)+O\left(x^{2}(\operatorname{Lnx})^{3}\right) \in \mathcal{L}
$$

and, after simplification:

$$
(\operatorname{Ln} x+1) \alpha_{0}=-x+O\left(x^{2}(\operatorname{Ln} x)^{3}\right) \in \mathcal{L}
$$

and finally:

$$
\alpha_{0}=-x(\operatorname{Ln} x)^{-1} \frac{1+\cdots}{1+\cdots} \in \mathcal{F}_{0}
$$

To prove the result for $\varphi_{k}, k \geq 1$, we proceed by recurrence on $k$. Suppose that the result is true for some $k \geq 0$. Remark that:

$$
\varphi_{k+1}(x)=\frac{d \varphi_{k}}{d x}(x) \cdot \frac{d x}{d \alpha_{1}}(x)
$$


From Lemma 2.3, it follows that $\frac{d x}{d \alpha_{1}} \in \mathcal{F}_{0}$ and that $\frac{d x}{d \alpha_{1}} \sim-x(\operatorname{Ln} x)^{2}$. Also from the recurrence hypothesis $\varphi_{k} \in \mathcal{F}_{0}$ and $\varphi_{k}(x) \sim(-1)^{k+1}$ $x(\operatorname{Ln} x)^{2 k-1}$. Applying again the Lemma 2.3 we deduce from this:

$$
\frac{d \varphi_{k}}{d x} \in \mathcal{F}_{0} \text { and } \frac{d \varphi_{k}}{d x} \sim(-1)^{k+1} P\left(L^{\prime}\right) \text { with } L=x(\text { Lnx })^{2 k-1}
$$

and

$$
P\left(L^{\prime}\right)=(\operatorname{Ln} x)^{2 k-1} \text {. }
$$

Then, $\frac{d x}{d \alpha_{1}} \cdot \frac{d \varphi_{k}}{d x} \in \mathcal{F}_{0}$ and is equivalent to $(-1)^{k+2} x(\operatorname{Ln} x)^{2 k+1}$, as we want.

From $\alpha_{1}(x) \sim(\operatorname{Ln} x)^{-1}$ we deduce that $\alpha_{1}(x)$ is invertible and from (2.2) that the inverse function:

$$
x\left(\alpha_{1}\right) \sim e^{-1} e^{1 / \alpha_{1}}\left(\text { for } \alpha_{1} \rightarrow 0_{-}\right) .
$$

Next, one can write:

$$
\frac{d^{k} \alpha_{0}}{d \alpha_{1}^{k}}\left(\alpha_{1}\right)=\varphi_{k}\left(x\left(\alpha_{1}\right)\right)
$$

Using the equivalence (2.14) and (2.20), one has:

$$
\frac{d^{k} \alpha_{0}}{d \alpha_{1}^{k}}\left(\alpha_{1}\right) \sim(-1)^{k+1} e^{-1} \alpha_{1}^{1-2 k} e^{1 / \alpha_{1}}\left(\text { for } \alpha_{1} \rightarrow 0_{-}\right)
$$

This justifies the fact that the equivalence $\alpha_{0} \sim-e^{-1} \alpha_{1} e^{1 / \alpha_{1}}$ "can be differentiated indefinitely" as it is claimed in Theorem 1.2.

\section{Smooth flatness in bifurcation diagrams of homoclinic connections (Theorem 1.1)}

We consider now a generic 2-parameter $\mathcal{C}^{\infty}$ unfolding $X_{\alpha}$ of some homoclinic connection $\Gamma$. We can suppose that the saddle $s$ and its local invariant manifolds are fixed in some system of coordinates. As explained in the introduction, one can suppose that the parameter is $\alpha=\left(\alpha_{0}, \alpha_{1}\right)$, where $\alpha_{1}=1-r, r$ being the ratio $\frac{\lambda_{1}(\alpha)}{\lambda_{2}(\alpha)}$ and $\alpha_{0}$ is the distance between the separatrices of $X_{\alpha}$ near the connection $\Gamma$.

The return map $P_{\alpha}(x)$ of $X_{\alpha}$ along $\Gamma$, is defined on some transverse segment $\sigma$, parametrized by $x \in[0, X[$ ( 0 , on the local stable separatrix 
of $s$ ) with range in some interval $\sigma^{\prime}$, parametrized by $]-X^{\prime}, X^{\prime}[$ with $X^{\prime}>X$.

Let be $\delta_{\alpha}(x)=\delta(x, \alpha)=P_{\alpha}(x)-x$, which maps $\sigma \times U$ into $\mathbf{R}$, where $U$ is some neighborhood of 0 in the parameter space. It was proved in $[\mathbf{R}]$ that $\delta_{\alpha}$ admits $(x, \omega)$-expansions at any order $\ell$. This means that if one defines $\omega\left(x, \alpha_{1}\right)=\frac{x^{-\alpha_{1}}-1}{\alpha_{1}}$ for $\alpha_{1} \neq 0$ and $\omega(x, 0)=-\operatorname{Ln} x$, there exists local $\mathcal{C}^{\infty}$ functions $a_{i j}(\alpha), 0 \leq j \leq i$ at $0 \in \mathbf{R}^{2}$ such that for any $\ell$ and for $(x, \alpha) \in] 0, X\left[\times U_{\ell}\left(U_{\ell} \subset U\right.\right.$ some neighborhood of $\left.0 \in \mathbf{R}^{2}\right)$ one has:

$$
\delta(x, \alpha)=\sum_{0 \leq j \leq i \leq \ell+1} a_{i j}(\alpha) x^{i} \omega^{j}+\Phi_{\ell}(x, \alpha)
$$

where $\Phi_{\ell}$ is $\mathcal{C}^{\ell}$ in $(x, \alpha)$ and $\ell$-flat at $x=0$ for any $\alpha$.

Moreover $\alpha_{00}(\alpha)=\alpha_{0}, \alpha_{11}(\alpha)=\alpha_{1}$ and $\bar{\alpha}_{2}=\alpha_{2}(0,0)$ such that $0<\bar{\alpha}_{2}<1$ if the saddle connection is unstable (i.e. if $\frac{d P_{0}}{d x}(0)>1$ ). This is supposed, from now on.

The expansion (3.1) looks rather similar to the expansion (2.1) for abelian integrals. The difference is that $-\operatorname{Ln} x$ is replaced now by the function $\omega$ which depends on $\alpha_{1}$. This fact will make the proofs more complicated because one will have to consider the derivatives of $\omega$ in $\alpha_{1}$. Nevertheless the proofs in this paragraph will follow the same general lines as in the previous paragraph.

Of course, it is rather easy to prove that one has a line $D$ in the parameter plane for the double limit cycles (which correspond to double zeros of $\delta)$ [DRS1]. This line is a graph of a function $\alpha_{0}\left(\alpha_{1}\right)$ for $\alpha_{1} \leq 0$ which can be parametrized by the value $x \in[0, X$ [ of the double zero: $D=\left\{\left(\alpha_{0}(x), \alpha_{1}(x)\right) \mid x \in[0, X[\}\right.$, taking $X>0$ sufficiently small. As in paragraph 2, we want to study the functions $\alpha_{0}(x), \alpha_{1}(x)$ before proving Theorem 2.1 for $\alpha_{0}\left(\alpha_{1}\right)=\alpha_{0}\left(x\left(\alpha_{1}\right)\right)$ where $x\left(\alpha_{1}\right)$ will be the local inverse of $\alpha_{1}(x)$. The functions $\alpha_{0}(x)$ and $\alpha_{1}(x)$ are $\mathcal{C}^{\infty}$ for $x \neq 0$ and we want to study them for $x$ near zero. For small values of $x, \alpha_{0}(x), \alpha_{1}(x)$ are given implicitly by the equations:

$$
\left\{\begin{array}{l}
\delta(x, \alpha)=\alpha_{0}+\alpha_{1} x \omega+\alpha_{2}(\alpha) x+\cdots=0 \\
\frac{\partial \delta}{\partial x}(x, \alpha)=\alpha_{1}\left(\left(1-\alpha_{1}\right) \omega-1\right)+\alpha_{2}(\alpha)+\cdots=0 .
\end{array}\right.
$$

Here and in the sequence: $+\cdots$ means some $(x, \omega)$-expansion in monomials $x^{i} \omega^{j}, i, j \in \mathbf{Z}$ with smooth coefficients in the parameter, plus a $\mathcal{C}^{\ell}$ flat remaining term as in the formula (3.1). All the monomials are of 
order of flatness strictly greater then the last term to the left of the $\operatorname{sign}+$.

The monomials will always be ordered in increasing order of flatness: $x^{i} \omega^{j}$ is flatter than $x^{i^{\prime}} \omega^{j^{\prime}} \Longleftrightarrow i>i^{\prime}$ or $i=i^{\prime}$ and $j<j^{\prime}$.

In formulas (3.2), the first line is (3.1) and the second one contains monomials $x^{i} \omega^{j}$ with $j \leq i+1$.

To obtain the asymptotics for $\alpha_{0}(x), \alpha_{1}(x)$ it is more convenient to rewrite $\delta$ as:

$$
\delta(x, \alpha)=\alpha_{0}+x^{1-\alpha_{1}}+\left(\alpha_{2}(\alpha)-1\right) x+O\left(x^{2} \omega^{2}\right)
$$

and:

$$
\frac{\partial \delta}{\partial x}(x, \alpha)=\left(1-\alpha_{1}\right) x^{-\alpha_{1}}+\left(\alpha_{2}-1\right)+o(1) .
$$

The first idea is to notice that some interesting functions remain bounded all along $D$ :

Lemma 3.1. The functions $x^{-\alpha_{1}(x)}, \alpha_{1}(x) \operatorname{Ln} x$ and $\alpha_{1}(x) \omega\left(x, \alpha_{1}(x)\right)$ are continuous at $x=0$ and more precisely:

(1) $x^{-\alpha_{1}(x)} \longrightarrow 1-\bar{\alpha}_{2}$ for $x \longrightarrow 0_{+}$,

(2) $-\alpha_{1}(x) \omega\left(x, \alpha_{1}(x)\right) \longrightarrow \bar{\alpha}_{2}$ for $x \longrightarrow 0_{+}$,

(3) $\alpha_{1}(x) \operatorname{Ln}(x) \longrightarrow v_{0}=-\operatorname{Ln}\left(1-\bar{\alpha}_{2}\right)$ for $x \longrightarrow 0_{+}$.

Proof: The point (1) follows from equation (3.4). Next $\alpha_{1} \omega=x^{-\alpha_{1}}-1$ and (1) implies (2). To prove the point (3) we introduce the analytic function:

$$
\Phi(u)=\frac{1-e^{-u}}{u} \text { for } u \neq 0, \quad \Phi(0)=1 .
$$

One has:

$$
\omega\left(x, \alpha_{1}\right)=-\operatorname{Ln} x \Phi\left(\alpha_{1} \operatorname{Ln} x\right) .
$$

Now, let be $\psi(u)=u \Phi(u)=1-e^{-u}$.

(3.6) $\psi$ maps diffeomorphically $\left[0, \infty\left[\right.\right.$ on $\left[0,1\left[\right.\right.$ and one has $\bar{\alpha}_{2}=\psi\left(v_{0}\right)$.

From (3.5), one obtains that:

$$
\alpha_{1} \omega=-\psi\left(\alpha_{1} \operatorname{Lnx}\right) .
$$

So, it follows by continuity that:

$$
\alpha_{1}(x) \operatorname{Ln} x \longrightarrow \psi^{-1}\left(\bar{\alpha}_{2}\right)=v_{0} .
$$

First, one can solve the first equation of (3.2) to obtain $\alpha_{0}$ as a function $\widetilde{\alpha}_{0}\left(x, \alpha_{1}\right)$. The proof is completely similar to the one of Proposition 2.1. One has just to replace $-\operatorname{Ln} x$ by $\omega$. So we just give the result: 
Proposition 3.2. The equation $\delta=0$ defines a function $\alpha_{0}=\widetilde{\alpha}_{0}\left(x, \alpha_{1}\right)$ which admits an $(x, \omega)$-expansion at any order $\ell$ :

$$
\widetilde{\alpha}_{0}=\sum_{0 \leq j \leq i \leq \ell+1} \beta_{i j}\left(\alpha_{1}\right) x^{i} \omega^{j}+\Phi_{\ell}\left(x, \alpha_{1}\right)
$$

where the $\beta_{i j}$ are local smooth functions of $\alpha_{1}$ and $\Phi_{\ell}$ is $\mathcal{C}^{\ell}$, $\ell$-flat at $x=0$ for any $\alpha_{1}$.

The beginning of the expansion of $\widetilde{\alpha}_{0}$, is given by:

$$
\widetilde{\alpha}_{0}\left(x, \alpha_{1}\right)=-\alpha_{1} x \omega-\alpha_{2}\left(\alpha_{1}, 0\right) x+\cdots
$$

Now, one can substitute $\alpha_{0}=\widetilde{\alpha}_{0}\left(x, \alpha_{1}\right)$ in the second equation of (3.2) to obtain an implicit equation for $\alpha_{1}$ :

$$
\delta_{1}\left(x, \alpha_{1}\right)=\frac{\partial \delta}{\partial x}\left(x, \widetilde{\alpha}_{0}\left(x, \alpha_{1}\right), \alpha_{1}\right)=0 .
$$

The function $\delta_{1}$ admits again $(x, \omega)$-expansions but now with $j \leq i+1$. The first terms in $\delta_{1}$ are:

$$
\delta_{1}\left(x, \alpha_{1}\right)=\alpha_{1}\left(\left(1-\alpha_{1}\right) \omega-1\right)+\alpha_{2}\left(\alpha_{1}, 0\right)+\cdots
$$

where $+\cdots$ contains monomials $x^{i} \omega^{j}$ with $i \geq 1$ and $j \leq i+1$.

In the proof of Lemma 3.1 one has noticed that $\omega\left(x, \alpha_{1}\right)=$ $-\operatorname{Ln} x \Phi\left(\alpha_{1} \operatorname{Lnx}\right)$. Moreover, along the line $D, \alpha_{1} \operatorname{Lnx}$ and also $\Phi\left(\alpha_{1} \operatorname{Lnx}\right)$ are bounded. From this it follows that $\frac{\omega\left(x, \alpha_{1}(x)\right)}{-L n x} \longrightarrow \frac{\bar{\alpha}_{2}}{v_{0}} \neq 0$ for $x \longrightarrow 0_{+}$. This gives the basic idea for the proof: to replace $\omega$ by $-\operatorname{Lnx}$. Of course to do this, we will have to introduce smooth functions of $\alpha_{1} \operatorname{Lnx}$ as coefficients.

Definition 3.1. $\mathcal{L}^{\omega}$ is the $\operatorname{ring}$ of $\mathcal{C}^{\infty}$ functions defined near 0 in $] 0, X[$ with the following properties. For any $f \in \mathcal{L}^{\omega}$ and any order $k$ one can write:

$$
f(x)=F_{k}\left(x, \alpha_{1}(x), \alpha_{1}(x) \operatorname{Ln} x\right)+\Phi_{k}\left(x, \alpha_{1}(x), \alpha_{1}(x) \operatorname{Ln} x\right)
$$

for $x \in] 0, X_{k}$ [ for some $X_{k}, 0<X_{k}<X$, where:

(1) $F_{k}(x, u, v)$ is a polynomial in $x^{ \pm 1},(\operatorname{Ln} x)^{ \pm 1}$ with smooth coefficients in $(u, v)$ defined in some neighborhood $V_{k}$ of $\left(0, v_{0}\right)$. Moreover $F_{k}$ on $] 0, X\left[\times V_{k+1}\right.$ is equal to the trunquation of $F_{k+1}$.

(2) $\Phi_{k}(x, u, v)$ is a $\mathcal{C}^{k}$ function on $] 0, X_{k}\left[\times V_{k}, k\right.$-flat at $x=0$ for any $(u, v) \in V_{k}$. 
Let $\mathcal{L}_{0}^{\omega}$, be the subset of functions in $\mathcal{L}^{\omega}$ such that leading term of $F_{k}$ (independent of $k) L(x)=x^{i}(\operatorname{Lnx})^{j}$ is different from $1(i$ or $j \neq 0)$, with a coefficient $a(u, v)$ verifying $a\left(0, v_{0}\right) \neq 0$.

We will also consider $\mathcal{F}^{\omega}$, the ring of fractions $f / g$ with $f \in \mathcal{L}^{\omega}$ and $g \in \mathcal{L}_{0}^{\omega}$. Any element of $\mathcal{F}^{\omega}$ can be written $\frac{f}{g}$ with $g=1+\cdots \in \mathcal{L}^{\omega}$. We define $\mathcal{F}_{0}^{\omega} \subset \mathcal{F}^{\omega}$ as the set of fractions $\frac{f}{g}$ with $f \in \mathcal{L}_{0}^{\omega}$ and $g=1+\cdots \in$ $\mathcal{L}^{\omega}$.

Remark. Any $h \in \mathcal{F}_{0}^{\omega}$ can be written $h(x)=a\left(\alpha_{1}, \alpha_{1} \operatorname{Lnx}\right) L \frac{f}{g}$ with $L=x^{i}(\operatorname{Lnx})^{j} \not \equiv 1$ and $f=1+\cdots, g=1+\cdots \in \mathcal{L}^{\omega}$. It is clear that $h(x) \sim a\left(0, v_{0}\right) L(x)$ and so that, $a\left(0, v_{0}\right)$ does not depend on the choices $f, g, \ldots$ The monomial $x^{i}(\operatorname{Ln} x)^{j}$ will be called the order of $h \in \mathcal{F}_{0}^{\omega}$.

The sets $\mathcal{F}^{\omega}, \mathcal{F}_{0}^{\omega}$ have the following elementary properties:

1) if $h_{1}, h_{2} \in \mathcal{F}_{0}^{\omega}$ have different orders, then $h_{1}+h_{2} \in \mathcal{F}_{0}^{\omega}$,

2) $h \in \mathcal{F}_{0}^{\omega} \Longleftrightarrow \frac{1}{h} \in \mathcal{F}_{0}^{\omega}$,

3) $h_{1}, h_{2} \in \mathcal{F}^{\omega} \Longrightarrow h_{1} h_{2} \in \mathcal{F}^{\omega}$.

The behaviour of $\mathcal{F}_{0}^{\omega}$ for the differentiation $\frac{d}{d x}$ is less easy to obtain.

We first look at some particular cases:

Lemma 3.3. $\alpha_{1}(x) \in \mathcal{F}_{0}^{\omega}$ and:

$$
\alpha_{1}(x)=\frac{\alpha_{2}\left(\alpha_{1}, 0\right)}{\left.\left(1-\alpha_{1}\right) \Phi\left(\alpha_{1} \operatorname{Ln} x\right)\right)}(\operatorname{Ln} x)^{-1} \frac{1+\cdots}{1+\cdots}
$$

(which gives again that $\left.\alpha_{1}(x) \sim v_{0}(\operatorname{Lnx})^{-1}\right)$.

Proof: This is a direct consequence of the expansion (3.10) of $\delta_{1}$. If we replace $\omega$ by $-\operatorname{Ln} x \Phi\left(\alpha_{1} \operatorname{Lnx}\right)$ in $+\cdots$, and then solve in $\alpha_{1}$, one obtains:

$$
\alpha_{1}=-\alpha_{2}\left(\alpha_{1}, 0\right) \omega^{-1} \frac{u}{1-\alpha_{1}-\omega^{-1}} \text { with } u=1+\cdots \in \mathcal{L}^{\omega}
$$

and finally:

$$
\alpha_{1}=\frac{\alpha_{2}\left(\alpha_{1}, 0\right)}{\left(1-\alpha_{1}\right) \Phi\left(\alpha_{1} \operatorname{Lnx}\right)}(\operatorname{Lnx})^{-1} \frac{u}{v}
$$

with $v=1+\cdots \in \mathcal{L}^{\omega}$

Lemma 3.4. $\alpha_{0}(x) \in \mathcal{F}_{0}^{\omega}$ and:

$$
\alpha_{0}(x)=c\left(\alpha_{1}, \alpha_{1} \operatorname{Ln} x\right) x(\operatorname{Ln} x)^{-1} \frac{1+\cdots}{1+\cdots} .
$$


with:

$$
c\left(\alpha_{1}, \alpha_{1} \operatorname{Ln} x\right)=-\frac{\alpha_{2}\left(\alpha_{1}, 0\right)\left(1-\alpha_{2}\left(\alpha_{1}, 0\right)\right)}{\left(1-\alpha_{1}\right)^{2} \Phi\left(\alpha_{1} \operatorname{Ln} x\right)}
$$

(so that $\alpha_{0}(x) \sim c\left(0, v_{0}\right) x(\operatorname{Ln} x)^{-1}$ with $\left.c\left(0, v_{0}\right)=-\left(1-\bar{\alpha}_{2}\right) v_{0}\right)$.

Proof: From the equation (3.8) for $\alpha_{0}$ and the Lemma 3.3, it follows easily that $\alpha_{0} \in \mathcal{F}^{\omega}$. To prove that $\alpha_{0} \in \mathcal{F}_{0}^{\omega}$, we bring the expression:

$$
-\alpha_{1} \omega=\frac{\alpha_{2}\left(\alpha_{1}, 0\right)-\alpha_{1}}{1-\alpha_{1}}+\cdots
$$

coming from (3.10) in the expression:

$$
\widetilde{\alpha}_{0}=-\alpha_{1} x \omega-\alpha_{2}\left(\alpha_{1}, 0\right) x+\cdots
$$

We obtain that:

$$
\begin{aligned}
& \widetilde{\alpha}_{0}=\frac{\alpha_{2}\left(\alpha_{1}, 0\right)-\alpha_{1}}{1-\alpha_{1}} x-\alpha_{2}\left(\alpha_{1}, 0\right) x+\cdots \\
& \widetilde{\alpha}_{0}=\frac{-\alpha_{1}\left(1-\alpha_{2}\left(\alpha_{1}, 0\right)\right)}{1-\alpha_{1}} x+\cdots
\end{aligned}
$$

The expression (3.13) is not an expression in $\mathcal{F}_{0}^{\omega}$ of order $(1,0)$ because the coefficient of the leading term is zero for $\alpha_{1}=0$. To obtain the expression (3.12), it suffices to substitute the term $\alpha_{1}$ in this coefficient by its expansion (3.11). (We do not modify the other occurrences of $\alpha_{1}$ in $(3.13))$.

Lemma 3.5. $\frac{d \alpha_{1}}{d x} \in \mathcal{F}_{0}^{\omega}$ and $\frac{d \alpha_{1}}{d x}(x) \sim-v_{0} x^{-1}(\operatorname{Lnx})^{-2}$.

Remark. The equivalence for $\frac{d \alpha_{1}}{d x}(x)$ is just given by the formal derivation of the equivalence $\alpha_{1}(x) \sim v_{0}(\operatorname{Ln} x)^{-1}$.

Proof: The equation (3.10) for $\alpha_{1}$ writes:

$$
\alpha_{1}\left(\left(1-\alpha_{1}\right) \omega-1\right)=-\alpha_{2}\left(\alpha_{1}, 0\right)+\cdots
$$

Let $Q\left(x, \alpha_{1}\right)$ be the right hand member of (3.14). It is a function in $\mathcal{L}_{0}^{\omega}$. By differentiation in $x$, we obtain:

$$
\begin{aligned}
{\left[\left(1-\alpha_{1}\right) \omega-1-\alpha_{1} \omega+\alpha_{1}\left(1-\alpha_{1}\right)\right.} & \left.\frac{\partial \omega}{\partial \alpha_{1}}-\frac{\partial Q}{\partial \alpha_{1}}\right] \frac{d \alpha_{1}}{d x} \\
& =-\alpha_{1}\left(1-\alpha_{1}\right) \frac{\partial \omega}{\partial x}+\frac{\partial Q}{\partial x}
\end{aligned}
$$


We look first at the factor $G$ in front of $\frac{d \alpha_{1}}{d x}$. Because $\frac{\partial \omega}{\partial \alpha_{1}}=$ $-\Phi^{\prime}\left(\alpha_{1} \operatorname{Lnx}\right)(\operatorname{Lnx})^{2}$ and $\frac{\partial Q}{\partial \alpha_{1}}$ are in $\mathcal{L}^{\omega}$, the same holds for $G$. The leading term of $G$ is given by:

$$
-\left(\left(1-2 \alpha_{1}\right) \Phi+\frac{\alpha_{2}\left(\alpha_{1}, 0\right)}{\Phi} \Phi^{\prime}\right) \operatorname{Ln} x .
$$

(Here $\Phi(v)$ and $\Phi^{\prime}(v)=\frac{d \Phi}{d v}(v)$ must be evaluated at $\left.v=\alpha_{1} L n x\right)$.

The coefficient of this leading term is equal to $a\left(\alpha_{1}, \alpha_{1} \operatorname{Lnx}\right)$ with:

$$
a(u, v)=-\left((1-2 u) \Phi(v)+\alpha_{2}(u, 0) \frac{\Phi^{\prime}(v)}{\Phi(v)}\right) .
$$

We have:

$$
-a\left(0, v_{0}\right)=\Phi\left(v_{0}\right)+\frac{\bar{\alpha}_{2}}{\Phi\left(v_{0}\right)} \Phi^{\prime}\left(v_{0}\right)
$$

which gives:

$$
a\left(0, v_{0}\right)=-\left(1-\bar{\alpha}_{2}\right) \neq 0 .
$$

So, we have that $G \in \mathcal{L}_{0}^{\omega}$.

Look now at the right hand term of (3.15):

$$
F=-\alpha_{1}\left(1-\alpha_{1}\right) \frac{\partial \omega}{\partial x}+\frac{\partial Q}{\partial x} .
$$

One has:

$$
\frac{\partial \omega}{\partial x}=-\left(\alpha_{1} \Phi^{\prime} \operatorname{Ln} x+\Phi\right) x^{-1}
$$

If in this expression, we replace $\alpha_{1}$, coefficient of $\Phi^{\prime} \operatorname{Lnx}$ by its expansion (3.11), we obtain, taking in account that $\frac{\partial Q}{\partial x}=O\left((\operatorname{Lnx})^{2}\right)$ :

$$
F=\frac{\alpha_{2}\left(\alpha_{1}, 0\right)}{\Phi}\left(\alpha_{2}\left(\alpha_{1}, 0\right)\left(1-\alpha_{1}\right) \Phi \Phi^{\prime}+\frac{\psi_{1}}{\psi_{2}} \Phi\right) x^{-1}(\operatorname{Ln} x)^{-1} \frac{\varphi_{1}}{\varphi_{2}}
$$

where $\varphi_{1}=1+\cdots, \varphi_{2}=1+\cdots \in \mathcal{L}^{\omega}$ as well as $\psi_{1}, \psi_{2}$.

Finally, using (3.17) and (3.21), one obtain the expression for $\frac{d \alpha_{1}}{d x}$ as a function of $\mathcal{F}_{0}^{\omega}$ :

$$
\frac{d \alpha_{1}}{d x}=\frac{F}{G}=t\left(\alpha_{1}, \alpha_{1} \operatorname{Ln} x\right) x^{-1}(\operatorname{Ln} x)^{-2} \frac{1+\cdots}{1+\cdots}
$$


with:

$$
t\left(\alpha_{1}, \alpha_{1} \operatorname{Lnx}\right)=-\frac{\alpha_{2}\left(\alpha_{1}, 0\right)}{\Phi} \frac{\left(1-\alpha_{1}\right) \alpha_{2} \frac{\Phi^{\prime}}{\Phi}+\Phi}{\left(1-2 \alpha_{1}\right) \Phi+\frac{\alpha_{2}}{\Phi} \Phi^{\prime}}
$$

We find that $t\left(0, v_{0}\right)=-v_{0}$, which gives:

$$
\frac{d \alpha_{1}}{d x} \sim-v_{0} x^{-1}(\operatorname{Ln} x)^{-2}
$$

We can now consider a general function in $\mathcal{F}_{0}^{\omega}$ :

Proposition 3.6. Let be $f(x)=c\left(\alpha_{1}, \alpha_{1} L n x\right) x^{i}(\operatorname{Lnx})^{j} \frac{1+\cdots}{1+\cdots}$ a function in $\mathcal{F}_{0}^{\omega}\left(\right.$ i.e.: $c\left(0, v_{0}\right) \neq 0$ ) and such that $i \neq 0$. Then $\frac{d f}{d x} \in \mathcal{F}_{0}^{\omega}$ and:

$$
\frac{d f}{d x}=i c\left(\alpha_{1}, \alpha_{1} \operatorname{Ln} x\right) x^{i-1}(\operatorname{Ln} x)^{j} \frac{1+\cdots}{1+\cdots} .
$$

Remark. Again, the equivalence for $\frac{d f}{d x}$ is just the formal differentiation of the equivalence $f(x) \sim c\left(0, v_{0}\right) x^{i}(\operatorname{Ln} x)^{j}$.

Take any function $g(x) \in \mathcal{L}^{\omega}$. At any order $\ell$, there exists a function $G(x, u, v)$ (we omit $\ell$ !) such that $g(x)=G\left(x, \alpha_{1}(x), \alpha_{1} \operatorname{Lnx}\right.$ ) where the dependence on $\alpha_{1}$ and $\alpha_{1} \operatorname{Lnx}$ is located in the smooth coefficients and the remaining term of the expansions introduced above in Definition 3.1. Now, we have:

$$
\frac{d g}{d x}=\frac{\partial G}{\partial x}+\alpha_{1} \frac{\partial G}{\partial v} x^{-1}+\left(\frac{\partial G}{\partial u}+\frac{\partial G}{\partial v} \operatorname{Lnx}\right) \frac{d \alpha_{1}}{d x} .
$$

Using this expression it is clear that $\frac{d g}{d x} \in \mathcal{L}^{\omega}$.

Now, we consider any $f(x) \in \mathcal{F}_{0}^{\omega}, f(x)=c\left(\alpha_{1}, \alpha_{1} L n x\right) x^{i}(\operatorname{Lnx})^{j} \frac{g}{h}$, with $g=1+\cdots, h=1+\cdots \in \mathcal{L}^{\omega}$.

Because $g, h=1+0\left((\operatorname{Lnx})^{-1}\right)$ one has that:

$$
\frac{d g}{d x}, \frac{d h}{d x}=0\left(x^{-1}(L n x)^{-2}\right)
$$

and also that:

$$
\frac{d}{d x}\left(\frac{g}{h}\right)=x^{-1}(\operatorname{Ln} x)^{-2} \frac{\bar{g}}{1+\cdots}
$$


with $\bar{g}, 1+\cdots \in \mathcal{L}^{\omega}$.

Let us consider now the derivation of the principal part of $f$ :

$$
\begin{aligned}
& \frac{d}{d x}\left[c x^{i}(\operatorname{Ln} x)^{j}\right]=\frac{d}{d x}\left(x^{i}(\operatorname{Ln} x)^{j}\right) c+x^{i}(\operatorname{Ln} x)^{j} \frac{d}{d x} c\left(\alpha_{1}, \alpha_{1} \operatorname{Ln} x\right) \\
& \frac{d}{d x}\left(x^{i}(\operatorname{Ln} x)^{j}\right)=i x^{i-1}(\operatorname{Ln} x)^{j}+\cdots(\text { because } i \neq 0) \\
& \frac{d}{d x} c\left(\alpha_{1}, \alpha_{1} \operatorname{Ln} x\right)=\frac{d \alpha_{1}}{d x}\left(\frac{\partial c}{\partial u}+\frac{\partial c}{\partial v} \operatorname{Ln} x\right)+x^{-1} \alpha_{1} \frac{\partial c}{\partial v} .
\end{aligned}
$$

Using Lemmas 3.4, 3.5, one can write this function in $\mathcal{L}^{\omega}$ with leading term: $x^{-1}(\operatorname{Ln} x)^{-1}$. Comparing with (3.28), we see that $\frac{d}{d x}\left[c x^{i}(\operatorname{Ln} x)^{j}\right) \in$ $\mathcal{L}_{0}^{\omega}$ with leading term $x^{i-1}(\operatorname{Lnx})^{j}$ and coefficient $i c\left(\alpha_{1}, \alpha_{1} \operatorname{Lnx}\right)$.

$$
\frac{d}{d x}\left[c x^{i}(\operatorname{Ln} x)^{j}\right]=i c x^{i-1}(\operatorname{Ln} x)^{j}+\cdots
$$

If we return now to $\frac{d f}{d x}$, one has:

$$
\frac{d f}{d x}=\frac{d}{d x}\left(c x^{i}(\operatorname{Ln} x)^{j}\right) \frac{g}{h}+c x^{i}(\operatorname{Ln} x)^{j} \frac{d}{d x}\left(\frac{g}{h}\right) .
$$

And, it follows from (3.26) and (3.30) that $\frac{d f}{d x}$ can be written in $\mathcal{F}_{0}^{\omega}$ :

$$
\frac{d f}{d x}=i c\left(\alpha_{1}, \alpha_{1} \operatorname{Ln} x\right) x^{i-1}(\operatorname{Ln} x)^{j} \frac{1+\cdots}{1+\cdots} .
$$

Now, as in paragraph 2 , one introduces for any $k \geq 0$, the function $\varphi_{k}(x)=\frac{d^{k} \alpha_{0}}{d \alpha_{1}^{k}}\left(\alpha_{1}(x)\right)$.

One has $\varphi_{0}(x)=\alpha_{0}(x) \in \mathcal{F}_{0}^{\omega}$, and from 3.12:

$$
\varphi_{0}(x) \sim-\left(1-\bar{\alpha}_{2}\right) v_{0} x(\operatorname{Ln} x)^{-1} .
$$

For $k \geq 0$, the $\varphi_{k}$ are related by the recurrence relation:

$$
\varphi_{k+1}(x)=\frac{d \varphi_{k}}{d x}(x)\left[\frac{d \alpha_{1}}{d x}(x)\right]^{-1} .
$$

From this, we deduce the: 
Proposition 3.7. For any $k \geq 0, \varphi_{k} \in \mathcal{F}_{0}^{\omega}$ and:

$$
\varphi_{k}(x) \sim(-1)^{k+1}\left(1-\bar{\alpha}_{2}\right) v_{0}^{-k+1} x(\operatorname{Ln} x)^{2 k-1} .
$$

Proof: For $k=0$ one has already noticed that $\varphi_{0}=\alpha_{0}$ and (3.34) reduces to $(3.12)$.

Now, by recurrence, suppose that $\varphi_{k} \in \mathcal{F}_{0}^{\omega}$ and that $\varphi_{k}(x) \sim(-1)^{k+1}$ $\left(1-\bar{\alpha}_{2}\right) v_{0}^{-k+1} x(\operatorname{Ln} x)^{2 k-1}$.

From Proposition 3.6, one obtains that:

$$
\frac{d \varphi_{k}}{d x} \in \mathcal{F}_{0}^{\omega} \text { and } \frac{d \varphi_{k}}{d x}(x) \sim(-1)^{k+1}\left(1-\bar{\alpha}_{2}\right) v_{0}^{-k+1}(\operatorname{Ln} x)^{2 k-1} .
$$

From (3.5), one has that $\frac{d \alpha_{1}}{d x} \in \mathcal{F}_{0}^{\omega}$ and $\frac{d \alpha_{1}}{d x} \sim-v_{0} x^{-1}(\operatorname{Lnx})^{-2}$ and finally: $\varphi_{k+1} \in \mathcal{F}_{0}^{\omega}$ with:

$$
\varphi_{k+1} \sim(-1)^{k+2}\left(1-\bar{\alpha}_{2}\right) v_{0}^{-(k+1)+1} x(\operatorname{Ln} x)^{2(k+1)-1} .
$$

We can now finish the proof of Theorem 1.1.

From $\delta_{1}\left(x, \alpha_{1}\right)=\left(1-\alpha_{1}\right) x^{-\alpha_{1}}+\left(\alpha_{2}\left(\alpha_{1}, 0\right)-1\right)+0\left(x \omega^{2}\right)=0$ we deduce that:

$$
x^{-\alpha_{1}}\left(1+0\left(x^{1+\alpha_{1}} \omega^{2}\right)\right)=\frac{1-\alpha_{2}}{1-\alpha_{1}}=1-\bar{\alpha}_{2}+o(1) .
$$

This gives:

$$
x\left(\alpha_{1}\right) \sim e^{-1} e^{\frac{v_{0}}{\alpha_{1}}} .
$$

Recall that, inversely $\alpha_{1}(x) \sim v_{0}(\operatorname{Lnx})^{-1}$.

From this, one can deduce that:

$$
\frac{d^{k} \alpha_{0}}{d \alpha_{1}^{k}}\left(\alpha_{1}\right)=\varphi_{k}\left(x\left(\alpha_{1}\right)\right) \sim(-1)^{k+1}\left(1-\bar{\alpha}_{2}\right) e^{-1} \frac{v_{0}^{k}}{\alpha_{1}^{2 k-1}} e^{v_{0} / \alpha_{1}}
$$

As it was announced in Theorem 1.1, this means that one can differentiate indefinitely the equivalence:

$$
\alpha_{0}\left(\alpha_{1}\right) \sim-\left(1-\bar{\alpha}_{2}\right) e^{-1} \alpha_{1} e^{v_{0} / \alpha_{1}}
$$




\section{Smooth flatness in bifurcation diagrams for perturbations of hamiltonian vector fields (Theorem 1.3)}

We consider now a perturbation of hamiltonian vector fields. In the proof of Theorem 1.3, one can replace the parametrization by the hamiltonian value $h$, by any smooth parametrization $x \in[0, X[$ like in paragraph 3.

In the present case the 2 parameters $\alpha_{0}, \alpha_{1}$ can be divided by the perturbation parameter $\varepsilon$ : $\alpha_{0}=\varepsilon \bar{\alpha}_{0}, \alpha_{1}=\varepsilon \bar{\alpha}_{1}$. Next, the function $\delta$ and its $(x, \omega)$-expansions at any order $\ell$, can also be divided by $\varepsilon$ :

$$
\delta\left(x, \bar{\alpha}_{0}, \bar{\alpha}_{1}, \varepsilon\right)=\varepsilon \bar{\delta}\left(x, \bar{\alpha}_{0}, \bar{\alpha}_{1}, \varepsilon\right) .
$$

The function $\bar{\delta}$ has also $(x, \omega)$-expansions at any order $\ell$ :

$$
\bar{\delta}\left(x, \bar{\alpha}_{0}, \bar{\alpha}_{1}, \varepsilon\right)=\bar{\alpha}_{0}+\bar{\alpha}_{1} x \omega+\bar{\alpha}_{2}\left(\bar{\alpha}_{0}, \bar{\alpha}_{1}, \varepsilon\right) x+\cdots
$$

One has $\bar{\alpha}_{2}(0,0,0) \neq 0$. We can suppose and we will suppose that $\bar{\alpha}_{2}(0,0,0)>0$. We consider $\omega$ as a function of $x, \bar{\alpha}_{1}, \varepsilon$ :

$$
\omega\left(x, \bar{\alpha}_{1}, \varepsilon\right)=\frac{x^{-\alpha_{1}}-1}{\alpha_{1}}=\frac{x^{-\varepsilon \bar{\alpha}_{1}}-1}{\varepsilon \bar{\alpha}_{1}} .
$$

We want to study the surface $D$ of double zeros for $\bar{\delta}$. It is defined by the equation:

$$
\left\{\begin{array}{l}
\bar{\delta}=0 \\
\frac{\partial \bar{\delta}}{\partial x}=\bar{\alpha}_{1}\left(\left(1-\varepsilon \bar{\alpha}_{1}\right) \omega-1\right)+\bar{\alpha}_{2}(\bar{\alpha})+\cdots=0 .
\end{array}\right.
$$

The surface $D$ is the graph of a function $\bar{\alpha}_{0}\left(\bar{\alpha}_{1}, \varepsilon\right)$ defined for $\varepsilon \geq 0$ and $\bar{\alpha}_{1} \leq 0$, small enough. This surface may be looked at as union of lines $D_{\varepsilon}$, graphs of functions: $\bar{\alpha}_{1} \rightarrow \bar{\alpha}_{0}\left(\bar{\alpha}_{1}, \varepsilon\right)$.

For each $\varepsilon \neq 0$ we find back the situation studied in the previous paragraph. So that $\bar{\alpha}_{0}\left(\bar{\alpha}_{1}, \varepsilon\right)$ is smoothly flat at $\bar{\alpha}_{1}=0$. For $\varepsilon=0$, we find back the situation studied in paragraph 2 and $\bar{\alpha}_{0}\left(\bar{\alpha}_{1}, 0\right)$ is also smoothly flat at $\bar{\alpha}_{1}=0$. Here, we want establish the flat smoothness along the line $\left\{\bar{\alpha}_{1}=0\right\}$ in the $\left(\varepsilon, \bar{\alpha}_{1}\right)$-plane for the 2 -variable function $\bar{\alpha}_{0}\left(\bar{\alpha}_{1}, \varepsilon\right)$. Because $\bar{\alpha}_{0}\left(\bar{\alpha}_{1}, \varepsilon\right)$ is smooth for $\bar{\alpha}_{1} \neq 0$, it will suffice to prove that any partial derivative:

$$
\partial^{i, j} \bar{\alpha}_{0}=\frac{\partial^{i+j} \bar{\alpha}_{0}}{\partial \bar{\alpha}_{1}^{i} \partial \varepsilon^{j}}
$$


goes to zero, uniformly in $\varepsilon$, for $\bar{\alpha}_{1} \longrightarrow 0$.

As in the previous paragraph, we can parametrize $D$ by $x \in[0, X[$, the position of the double zero: $D=\left\{\left(\bar{\alpha}_{0}(x, \varepsilon), \bar{\alpha}_{1}(x, \varepsilon)\right) \mid x \in[0, X[, \varepsilon\right.$ small enough\}.

The function $\bar{\alpha}_{1}(x, \varepsilon)$ has an inverse function $x\left(\bar{\alpha}_{1}, \varepsilon\right)$ and $\bar{\alpha}_{0}\left(\bar{\alpha}_{1}, \varepsilon\right)$ is equal to $\bar{\alpha}_{0}\left(x\left(\bar{\alpha}_{1}, \varepsilon\right), \varepsilon\right)$.

Also, like in paragraph 3 , one introduces the $x$-parametrization for partial derivatives of $\bar{\alpha}_{0}\left(\bar{\alpha}_{1}, \varepsilon\right)$ :

$$
\varphi_{i j}(x, \varepsilon)=\partial^{i, j} \bar{\alpha}_{0}\left(\bar{\alpha}_{1}(x, \varepsilon), \varepsilon\right) .
$$

The smooth flatness of $\bar{\alpha}_{0}\left(\bar{\alpha}_{1}, \varepsilon\right)$ will follow from:

Proposition 4.1. For any $i, j \geq 0, \varphi_{i j}(x, \varepsilon)=O\left(x|\operatorname{Ln} x|^{2 i+j-1}\right)$ uniformly in $\varepsilon$ in the sense that there exist constants $M_{i j}>0$ such that:

$$
\left|\varphi_{i j}(x, \varepsilon)\right| \leq M_{i j} x|\operatorname{Ln} x|^{2 i+j-1}
$$

for all $x, \varepsilon$.

Before proving this proposition, we restate without proof the Lemma 3.1:

Lemma 4.2. Let be $\widetilde{\alpha}_{2}(\varepsilon)=\bar{\alpha}_{2}(0,0, \varepsilon)$. The functions $x^{-\varepsilon \bar{\alpha}_{1}}$, $\bar{\alpha}_{1} \omega\left(x, \bar{\alpha}_{1}, \varepsilon\right)$ and $\bar{\alpha}_{1} \operatorname{Ln} x$, with $\bar{\alpha}_{1}=\bar{\alpha}_{1}(x, \varepsilon)$, are continuous along $\{x=$ $0\}$.

More precisely:

(1) $x^{-\varepsilon \bar{\alpha}_{1}} \longrightarrow 1-\varepsilon \widetilde{\alpha}_{2}(\varepsilon)$

(2) $-\bar{\alpha}_{1} \omega \longrightarrow \widetilde{\alpha}_{2}(\varepsilon)$

(3) $\bar{\alpha}_{1} \operatorname{Ln}(x) \rightarrow \widetilde{v}_{0}(\varepsilon)=-\frac{\operatorname{Ln}\left(1-\varepsilon \widetilde{\alpha}_{2}(\varepsilon)\right)}{\varepsilon}=\widetilde{\alpha}_{2}(0)+0(\varepsilon)$ for $x \longrightarrow 0_{+}$, uniformly in $\varepsilon$.

Now, we can introduce sets of functions, similar to those in the previous paragraph:

Definition 4.1. $\mathcal{L}^{\omega, \varepsilon}$ is a ring of $\mathcal{C}^{\infty}$ functions, defined for $(x, \varepsilon)$ small and positive $(x>0, \varepsilon \geq 0)$. Apart from the dependence on $\varepsilon$, the definition is similar to the Definition 3.1: we just suppose that the coefficients in $F_{k}$ are smooth functions of $\bar{\alpha}_{1}, \bar{\alpha}_{1} \operatorname{Lnx}$ and $\varepsilon$ and that the rest $\Phi_{k}$ is $\mathcal{C}^{k}$ in $x, \bar{\alpha}_{1}, \bar{\alpha}_{1} \operatorname{Lnx}, \varepsilon$ and $k$-flat at $x=0$.

Let be $\mathcal{L}_{0}^{\omega, \varepsilon}$, the subset of functions in $\mathcal{L}^{\omega, \varepsilon}$ such that the leading term $L(x)=x^{i}(\operatorname{Ln} x)^{j}$ is different from $1(i$ or $j \neq 0)$, with a coefficient 
$a(u, v, s)$ such that $a\left(0, \widetilde{v}_{0}(\varepsilon), \varepsilon\right) \neq 0, \varepsilon$ small enough (it suffices that $\left.a\left(0, \widetilde{v}_{0}(0), 0\right) \neq 0\right)$.

$\mathcal{F}^{\omega, \varepsilon}$ will be the ring of fractions $f / g$ with $f \in \mathcal{L}^{\omega, \varepsilon}$ and $g \in \mathcal{L}_{0}^{\omega, \varepsilon}$, and $\mathcal{F}_{0}^{\omega, \varepsilon}$ the subset of $\mathcal{F}^{\omega, \varepsilon}$ of fractions $\frac{f}{g}$ with $f \in \mathcal{L}_{0}^{\omega, \varepsilon}$ and $g=1+\cdots \in$ $\mathcal{L}^{\omega, \varepsilon}$.

Remark. If $h=c\left(\alpha_{1}, \alpha_{1} \operatorname{Ln} x, \varepsilon\right) x^{i}(\operatorname{Ln} x)^{j} \frac{1+\cdots}{1+\cdots} \in \mathcal{F}_{0}^{\omega, \varepsilon}$ then $h(x, \varepsilon) \sim$ $c\left(0, \widetilde{v}_{0}(\varepsilon), \varepsilon\right) x^{i}(\operatorname{Lnx})^{j}$. The function $c\left(0, \widetilde{v}_{0}(\varepsilon), \varepsilon\right)$ is continuous in $\varepsilon$. To prove Proposition 4.1, it will be sufficient to prove that $\varphi_{i, j}(x, \varepsilon)$ belongs to $\mathcal{F}_{0}^{\omega, \varepsilon}$ with $x(\operatorname{Ln} x)^{2 i+j-1}$ as leading monomial.

Now the proofs of Lemmas 3.3, 3.4 and 3.5 can be easily generalized here. The only changes is that one has to replace the derivative $\frac{\partial \omega}{\partial \alpha_{1}}$ by $\frac{\partial \omega}{\partial \bar{\alpha}_{1}}=\frac{\partial \omega}{\partial \alpha_{1}} \cdot \varepsilon$. This implies that the variation of $\omega$ with $\bar{\alpha}_{1}$ is continuous at $\varepsilon=0$, where $\omega$ is replaced by $-\operatorname{Lnx}$.

So, one has that $\bar{\alpha}_{1}(x, \varepsilon), \bar{\alpha}_{0}(x, \varepsilon)$ and $\frac{d \bar{\alpha}_{1}}{d x}(x, \varepsilon)$ belong to $\mathcal{F}_{0}^{\omega, \varepsilon}$ with:

$$
\bar{\alpha}_{1}(x, \varepsilon) \sim \widetilde{v}_{0}(\varepsilon)(\operatorname{Lnx})^{-1}, \bar{\alpha}_{0}(x, \varepsilon) \sim-\left(1-\varepsilon \widetilde{\alpha}_{2}(\varepsilon)\right) \widetilde{v}_{0}(\varepsilon) x(L n x)^{-1}
$$

and:

$$
\frac{d \bar{\alpha}_{1}}{d x}(x, \varepsilon) \sim-\widetilde{v}_{0}(\varepsilon) x^{-1}(\operatorname{Ln} x)^{-2} .
$$

Moreover, if $f(x, \varepsilon)=c\left(\bar{\alpha}_{1}, \bar{\alpha}_{1} \operatorname{Ln} x, \varepsilon\right) x^{i}(\operatorname{Ln} x)^{j} \frac{1+\cdots}{1+\cdots}$ is a function in $\mathcal{F}_{0}^{\omega, \varepsilon}$, (i.e.: $\left.c\left(0, \widetilde{v}_{0}, 0\right) \neq 0\right)$ with $i \neq 0$, then $\frac{\partial f}{\partial x}(x, \varepsilon) \in \mathcal{F}_{0}^{\omega, \varepsilon}$ and:

$$
\frac{\partial f}{\partial x}=i c x^{i-1}(\operatorname{Ln} x)^{j} \frac{1+\cdots}{1+\cdots} .
$$

This implies that the equivalence $f(x, \varepsilon) \sim c(0, \widetilde{v}(\varepsilon), \varepsilon) x^{i}(\operatorname{Lnx})^{j}$ can be formally differentiated: $\frac{\partial f}{\partial x}(x, \varepsilon) \sim i c(0, \widetilde{v}(\varepsilon), \varepsilon) x^{i-1}(\operatorname{Ln} x)^{j}$.

By an argument similar to the one in paragraph 3 , these results imply that:

$$
\varphi_{i, 0}(x, \varepsilon) \in \mathcal{F}_{0}^{\omega, \varepsilon} \text { with an order } x(\operatorname{Ln} x)^{2 i-1} .
$$

Next, we have to consider derivatives in $\varepsilon$. We will say that $h \in \mathcal{F}^{\omega, \varepsilon}$ has an order $x^{i}(\operatorname{Ln} x)^{j}$ if it can be written $h=\frac{f}{g}$ with $g=1+\cdots \in$ $\mathcal{L}^{\omega, \varepsilon}$ and $f=a\left(\bar{\alpha}_{1}, \bar{\alpha}_{1} \operatorname{Lnx}, \varepsilon\right) x^{i}(\operatorname{Lnx})^{j}+\cdots$ with a smooth coefficient $a(u, v, \varepsilon)$ which may be equal to zero at $\left(0, \widetilde{v}_{0}, 0\right)$. Of course this order is not an intrinsic property of $h$ but depends on the choice of an expansion for $f$. 
Lemma 4.3. If $h \in \mathcal{F}^{\omega, \varepsilon}$ has an order $x^{i}(\operatorname{Lnx})^{j}$ then $\frac{\partial h}{\partial \varepsilon} \in \mathcal{F}^{\omega, \varepsilon}$ and has a same order.

Proof: We begin to prove this result for the function $\bar{\alpha}_{1}(x, \varepsilon)$. Like in paragraph 3 , we can solve the first line of (4.4) to obtain $\bar{\alpha}_{0}=\widetilde{\alpha}_{0}\left(x, \bar{\alpha}_{1}, \varepsilon\right)$ and next eliminate $\bar{\alpha}_{0}$ in the second line of (4.4) to obtain an implicit equation for $\bar{\alpha}_{1}(x, \varepsilon)$ :

$$
\begin{gathered}
\delta_{1}\left(x, \bar{\alpha}_{1}, \varepsilon\right)=\frac{\partial \delta}{\partial x}\left(x, \widetilde{\alpha}_{0}\left(x, \alpha_{1}, \varepsilon\right), \varepsilon\right)=0 \text { which writes: } \\
\bar{\alpha}_{1}\left(\left(1-\varepsilon \bar{\alpha}_{1}\right) \omega-1\right)=c\left(\bar{\alpha}_{1}, \varepsilon\right)+\cdots
\end{gathered}
$$

where $c\left(\bar{\alpha}_{1}, \varepsilon\right)=-\bar{\alpha}_{2}\left(\widetilde{\alpha}_{0}\left(0, \bar{\alpha}_{1}, \varepsilon\right), \bar{\alpha}_{1}, \varepsilon\right)$ is smooth with $c(0,0) \neq 0$.

Taking the derivative in $\varepsilon$ of (4.9) one obtains:

$$
\left[\left(1-2 \varepsilon \bar{\alpha}_{1}\right) \omega-1+\bar{\alpha}_{1}\left(1-\varepsilon \bar{\alpha}_{1}\right) \frac{\partial \omega}{\partial \bar{\alpha}_{1}}-\frac{\partial c}{\partial \bar{\alpha}_{1}}+\cdots\right] \frac{\partial \bar{\alpha}_{1}}{\partial \varepsilon}=O(1)
$$

where the right hand term of (4.10) is a function of $\mathcal{L}^{\omega, \varepsilon}$ of order 1 . Now, taking in account that $\bar{\alpha}_{1}(x, \varepsilon) \in \mathcal{F}_{0}^{\omega, \varepsilon}$ of order $(\operatorname{Lnx})^{-1}$, that $\omega\left(x, \bar{\alpha}_{1}, \varepsilon\right)=-\operatorname{Ln} x \Phi\left(\varepsilon \bar{\alpha}_{1} \operatorname{Lnx}\right)$ (where $\Phi(u)=\frac{1-e^{-u}}{u}$, for $u \neq 0$, like in paragraph 3$)$, it is easy to verify that the bracket, coefficient of $\frac{\partial \bar{\alpha}_{1}}{\partial \varepsilon}$ in (4.10), is in $\mathcal{L}_{0}^{\omega, \varepsilon}$ of order $\operatorname{Lnx}$. It follows that: $\frac{\partial \bar{\alpha}_{1}}{\partial \varepsilon} \in \mathcal{F}^{\omega, \varepsilon}$ with the same order $(\operatorname{Lnx})^{-1}$ as $\bar{\alpha}_{1}$.

Consider now some $f(x, \varepsilon)=c\left(\bar{\alpha}_{1}, \bar{\alpha}_{1} \operatorname{Lnx}, \varepsilon\right) x^{i}(\operatorname{Lnx})^{j}+\cdots$ in $\mathcal{L}^{\omega, \varepsilon}$ with order $x^{i}(\operatorname{Lnx})^{j}$, with $c(u, v, z)$ smooth.

One has that:

$$
\frac{\partial f}{\partial \varepsilon}=\left\{\left(\frac{\partial c}{\partial u}+\operatorname{Ln} x \frac{\partial c}{\partial v}\right) \frac{d \bar{\alpha}_{1}}{d \varepsilon}+\frac{\partial c}{\partial z}\right\} x^{i}(\operatorname{Ln} x)^{j}+\cdots
$$

Using the above result for $\frac{\partial \bar{\alpha}_{1}}{\partial \varepsilon}$, one obtains that $\frac{\partial f}{\partial \varepsilon} \in \mathcal{L}^{\omega, \varepsilon}$ of order $x^{i}(\operatorname{Ln} x)^{j}$.

Finally, let us consider $h=\frac{f}{g}$ with $f=c x^{i}(\operatorname{Lnx})^{j} \in \mathcal{L}^{\omega, \varepsilon}$ of order $x^{i}(\operatorname{Ln} x)^{j}$ and $g=1+\cdots \in \mathcal{L}^{\omega, \varepsilon}$ of order 1 :

$$
\frac{\partial h}{\partial \varepsilon}=\frac{\frac{\partial f}{\partial \varepsilon} g-\frac{\partial g}{\partial \varepsilon} f}{g^{2}}
$$

Because the order of $\frac{\partial f}{\partial \varepsilon}$ and $f$ is $x^{i}(\operatorname{Lnx})^{j}$ and the order of $g$ and $\frac{\partial g}{\partial \varepsilon}$ is 1 , one has that $\frac{\partial h}{\partial \varepsilon} \in \mathcal{F}^{\omega, \varepsilon}$ of order $x^{i}(\operatorname{Lnx})^{j}$. 
We can now finish the proof of Proposition 4.1. More precisely, we will prove inductively on $j$, that:

$$
\varphi_{i, j}(x, \varepsilon) \in \mathcal{F}^{\omega, \varepsilon} \text { of order } x(\operatorname{Lnx})^{2 i+j-1} .
$$

The equation (4.8) gives the result for $j=0$.

Suppose now that (4.13) was true for some $j \geq 0$.

If one differentiates in $\varepsilon$ the equation (4.5), one obtains:

$$
\varphi_{i, j+1}(x, \varepsilon)=\frac{\partial \varphi_{i j}}{\partial \varepsilon}(x, \varepsilon)-\varphi_{i+1, j} \frac{\partial \bar{\alpha}_{1}}{\partial \varepsilon} .
$$

Now, it follows from Lemma 4.3 and the induction hypothesis that each function in the right hand term of (4.14) is in $\mathcal{F}^{\omega, \varepsilon}$ and that $\varphi_{i+1, j}$ has order $x(L n x)^{2 i+j+1}, \frac{\partial \bar{\alpha}_{1}}{\partial \varepsilon}$ has order $(L n x)^{-1}$ and $\frac{\partial \varphi_{i j}}{\varphi \varepsilon}$ has order $x(\operatorname{Ln} x)^{2 i+j-1}$.

So finally, $\varphi_{i, j+1}(x, \varepsilon)$ is in $\mathcal{F}^{\omega, \varepsilon}$ with order $x(\operatorname{Ln} x)^{2 i+(j+1)-1}$. This concludes the proof of Proposition 4.1.

\section{References}

[D] F. Dumortier, Local study of planar vector fields: singularities and their unfoldings, in "Structure in Dynamics-Finite dimensional deterministic Studies," (H. W. Broer, F. Dumortier, S. J. Van Strien and F. Takens, eds.), Studies in Mathematical Physics 2, North Holland, 1991, pp. 161-241.

[DRS1] F. Dumortier, R. Roussarie and J. Sotomayor, Generic 3 -parameter families of planar vector fields on the plane, unfolding a singularity with nilpotent linear part. The cusp case, Ergordic Theory Dynam. Systems 7 (1987), 375-413.

[DRS2] F. Dumortier, R. Roussarie and J. Sotomayor, Generic 3-parameter families of planar vector fields, unfoldings of saddle, focus and elliptic singularities with nilpotent linear part, in "Bifurcation of Planar Vector Fields: Nilpotent Singularities and Abelian Integrals," (F. Dumortier and als., eds.), Lect. Notes in Math. 1480, Springer-Verlag, Berlin-Heidelberg-New-York, 1991, pp. 1-141.

[K] A. Khovanskiı, "Fewnomials," Amer. Math. Soc., Providence, RI, 1991.

[LR] J.-M. LION AND J.-P. RoLin, Théorème de préparation pour les fonctions logarithmico-exponentielles, Prépublication du Laboratoire de Topologie, Université de Bourgogne 98 (1996). 
[R] R. Roussarie, On the number of limit cycles which appear by perturbation of separatrix loop of planar vector fields, Bol. Soc. Brasil Mat. 17(2) (1989), 73-117.

1991 Mathematics subject classifications: 58F14.

Université de Bourgogne

Laboratoire de Topologie - U.M.R. 5584 du C.N.R.S.

U.F.R. des Sciences et Techniques

9, Avenue Alain Savary

B.P. 400

21011 Dijon Cedex

FRANCE

e-mail: roussari@u-bourgogne.fr

Primera versió rebuda el 30 de Novembre de 1996, darrera versió rebuda el 17 de Febrer de 1997 